\title{
A method for reducing mean flow in oscillating-grid turbulence
}

\author{
Mark W. McCorquodale ${ }^{1,2} \mathbb{D} \cdot$ R. J. Munro' ${ }^{1}$
}

Received: 3 August 2018 / Revised: 19 October 2018 / Accepted: 21 October 2018 / Published online: 9 November 2018 (c) The Author(s) 2018

\begin{abstract}
Oscillating-grid turbulence (OGT) is an experimental tool that has been widely used to study the role of turbulent fluctuations under conditions of small mean flow. We report experiments to investigate the structure of the turbulent flow produced by an oscillating grid, using velocity measurements obtained through the application of two-dimensional particle image velocimetry in the vertical plane through the centre of the grid. Ensemble averages of the fluid velocity measurements at specific stages of the grid's oscillation indicate that mean flow is induced in OGT by the merging of grid-induced jets close to the tank sidewalls. The installation of an open-ended 'inner box' (with its top edge positioned just below the bottom of the grid's oscillation) is shown to inhibit the merging of the jets, thereby resulting in a reduction in the magnitude of the mean flow within the interior of the inner box region. Measurements of the time-averaged root-mean-square turbulent velocity components and the time-averaged turbulent kinetic energy flux indicate that the installation of the inner box results in turbulence that is in good agreement with the well-established models of OGT across the central 50\% of the inner box's width, but that distinct anisotropic regions exist adjacent to the vertical sidewalls. We anticipate that this simple amendment to reduce the mean flow present in OGT can be readily used in future work that utilises OGT to isolate the effects of turbulent fluctuations from those of the mean flow.
\end{abstract}

\section{Graphical abstract}

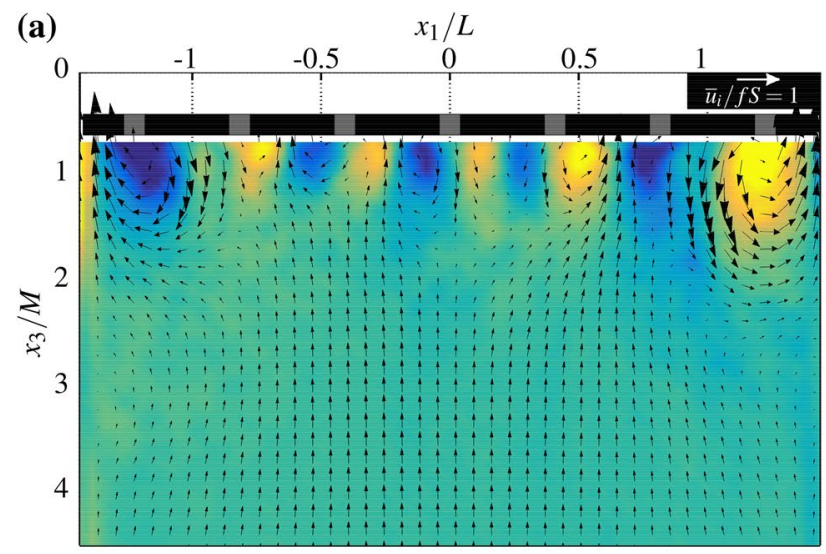

MWM acknowledges financial support through an Engineering and Physical Sciences Research Council Studentship (number 1510466).

Mark W. McCorquodale

m.w.mccorquodale@ reading.ac.uk

Extended author information available on the last page of the article

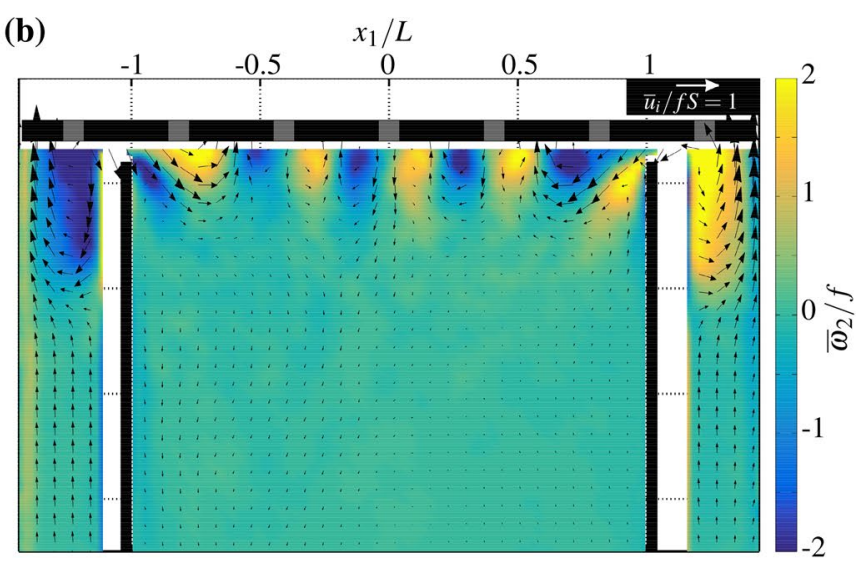

\section{Introduction}

Oscillating-grid turbulence (OGT) has been used extensively over the last 50 years as an experimental tool to study the role of turbulent fluctuations. The typical set-up consists of a planar grid, with uniform mesh spacing $M$, positioned horizontally inside a water-filled container and made to oscillate vertically with frequency $f$ and stroke $S$. 
The grid's oscillatory motion produces stationary turbulence that decays spatially in the grid-normal direction, but is approximately homogeneous in planes parallel to the grid, with mean-flow velocity components that are small in magnitude compared to the fluctuations. It is this approximate 'zero-mean-shear' property that has resulted in OGT being used to investigate a broad range of problems, which include the study of turbulent entrainment across a density interface (see, for example, Turner 1968; Hopfinger and Toly 1976; McGrath et al. 1997), particle coagulation and particle suspension (Brunk et al. 1998; Huppert et al. 1995; Wan Mohtar and Munro 2013), the growth and collapse of a turbulent front (Honey et al. 2014; De Silva and Fernando 1998), rotating turbulent flows (Hopfinger et al. 1982; Kinzel et al. 2009), and the inhibiting effect of boundaries on turbulence, including free surfaces (Brumley and Jirka 1987), density interfaces (Hannoun et al. 1988; Kit et al. 1997) and impermeable boundaries (Hannoun et al. 1988; McCorquodale and Munro 2017, 2018). The classical OGT apparatus has also inspired the development of new or related apparatus that produce a 'zero-mean-shear' turbulent flow through the use of, for example, multiple oscillating grids (Shy et al. 1997) or arrays of randomly firing jets (Variano et al. 2004).

The widespread use of OGT has resulted in the properties of the turbulence produced being studied extensively and a standard model to describe OGT has been developed. For a grid made of bar elements with square cross-section, the turbulence produced can be described by (Hopfinger and Toly 1976; Fernando and De Silva 1993)

$\left(\overline{u_{1}^{\prime 2}}\right)^{1 / 2}=C_{1} M^{1 / 2} S^{3 / 2} f x_{3}^{-1}$,

$\left(\overline{u_{3}^{\prime 2}}\right)^{1 / 2}=C_{2}\left(\overline{u_{1}^{\prime 2}}\right)^{1 / 2}$,

$\bar{\ell}=C_{3} x_{3}$,

where $x_{3}$ denotes the grid-normal direction, $\bar{\ell}$ is the timeaveraged integral length scale, and $\overline{\left(u_{1}^{\prime 2}\right)^{1 / 2}}, \overline{\left(u_{2}^{\prime 2}\right)^{1 / 2}}$ and $\left(\overline{u_{3}^{\prime 2}}\right)^{1 / 2}$ are the time-averaged root-mean-square (rms) velocity components. The symmetry of the grid means that $\left(\overline{u_{1}^{\prime 2}}\right)^{1 / 2} \approx\left(\overline{u_{2}^{\prime 2}}\right)^{1 / 2}$ is assumed. Typical values previously reported for the empirical coefficients are $C_{1}=0.2-0.5$, $C_{2}=1.1-1.4$ and $C_{3}=0.1-0.25$ (De Silva and Fernando 1994; Kit et al. 1997). This model was derived (for example, see Hopfinger and Toly 1976) from the transport equation for turbulent kinetic energy (TKE) under the simplifying assumptions of zero mean flow, homogeneity (in planes parallel to the grid) and negligible viscous transport. We stress, however, that the model is only valid sufficiently far from the grid (typically, for $x_{3} \gtrsim 2.5 M$ ). Close to the grid (i.e., for $\left|x_{3}\right| \lesssim 2.5 M$, henceforth referred to as the 'near-grid region' NGR), jets form in the wake of the grid elements resulting in a flow field in the NGR characterised by the presence of energetic, mesh size coherent vortex structures that interact and breakdown as they are advected away from the grid. However, beyond the NGR (i.e., for $x_{3} \gtrsim 2.5 M$, henceforth referred to as the 'turbulent-diffusive region' TDR), the jet structures have broken down and the flow is characterised by fluctuations that are large compared to mean-flow components, which decay in the grid-normal direction $\left(x_{3}\right)$ in a way that is well-approximated by Eq. (1).

However, that is not to say the mean flow is not an important factor to consider when using OGT (or when designing OGT apparatus). Although the mean velocity components are typically assumed to be small compared the rms fluctuations, the mean flow is not completely negligible. As a result, several studies have attempted to analyse the mean flow (Fernando and De Silva 1993; McKenna and McGillis 2004), resulting in a number of conditions on the oscillating-grid mechanism that should be satisfied to minimise the mean flow. The conditions specified include the use of a grid oscillation frequency $f$ less than $7 \mathrm{~Hz}$ (McDougall 1979) and a grid solidity less than $40 \%$ (Hopfinger and Toly 1976). Under these conditions Fernando and De Silva (1993) reported typical values for the 'mean flow intensity' (here used to denote the ratio of mean flow velocity component to the rms velocity component) of approximately 1.3 when using a grid constructed with a square perimeter edge, but that this ratio could be reduced to as low as 0.1 by designing the grid with edge conditions such that the tank side walls act as a plane of reflective symmetry to the grid. However, it has also been reported that even in studies that meet these criteria, including the reflective-symmetry condition of the grid design, that the ratio of mean flow intensity can approach unity and that the magnitude of the mean flow can vary by as much as $25 \%$ between consecutive experiments conducted under nominally identical conditions (McKenna and McGillis 2004).

Drayton (1993) proposed that a mean flow is induced in OGT when there is a significant difference in the relative strength of the jets produced by the oscillating grid in different regions of the tank, and suggested the Coanda effect to be the dominant mechanism for this. Drayton (1993) argued that, with OGT, a jet produced by the outermost grid elements is drawn onto the tank sidewall by the Coanda effect, preventing a symmetric growth of the jet about its axis. As a result, the jet's growth is inhibited in the wall-normal direction, with a comparatively greater spread of the jet in the wall-tangential direction, meaning that edge jets are more likely to merge with each other than jets produced closer to the centre of the grid. Drayton (1993) also proposed that the merged edge jets are more energetic than those produced close to the centre of the grid, and so do not necessarily breakdown within the NGR, giving rise to a more intense mean flow adjacent to the tank sidewalls, which results in 
large-scale circulations throughout the tank's interior. In the experiments reported by Drayton (1993), the merged edge jets were observed to have a wall-normal thickness of about two mesh lengths (i.e., $2 M$ ).

Previous studies (Hopfinger and Toly 1976; Dickinson and Long 1983) have suggested the use of an open-ended 'inner box', positioned symmetrically below the grid, can reduce large-scale circulations present in the tank that contribute significantly to the mean flow. Moreover, Drayton (1993) proposed (in the absence of supporting data) that the installation of an inner box could reduce large-scale circulations induced by the Coanda effect, but in order for the inner box to be effective in reducing the mean flow, it should be positioned with its top edge below the NGR (i.e., below $x_{3} \approx 2.5 M$ ), otherwise it was argued that the Coanda mechanism would also act within the inner box itself.

However, experimental data analysing how the presence of an inner box reduces mean flow, or the extent of any reduction, has not previously been reported. Therefore, this paper analyses the mean flow produced by OGT and shows how the installation of an inner box, positioned symmetrically below the grid, can be used to significantly reduce the mean flow present in OGT, such that turbulence produced is better described by the standard model (i.e., Eq. 1). In Sect. 2 we describe the OGT apparatus used and the application of particle image velocimetry (PIV) to acquire data sets for both the NGR and TDR. Measurements of the mean flow are presented in Sect. 3, in which ensemble averages are used to determine the statistical properties of the flow in the NGR, at different stages during the grid's oscillation, and time averages are used to determine the flow statistics in the TDR. Measurements of turbulent statistics are also presented, including the time-averaged rms velocity components $\left(\overline{u_{1}^{\prime 2}}\right)^{1 / 2}$ and $\left(\overline{u_{3}^{\prime 2}}\right)^{1 / 2}$, degree of isotropy $\left(\overline{u_{3}^{\prime 2}}\right)^{1 / 2} /\left(\overline{u_{1}^{\prime 2}}\right)^{1 / 2}$ and turbulent energy flux, which illustrate the effects of both tank side walls and mean flow intensity on the turbulence statistics. Conclusions are made in Sect. 4.

\section{Experiments}

\subsection{Apparatus \& set-up}

A schematic view of the experimental set-up is shown in Fig. 1, which is similar to those widely used in previous studies (see, for example, Thompson and Turner 1975; Hopfinger and Linden 1982). The experiments were conducted in a transparent container with internal dimensions $35.2 \mathrm{~cm} \times 35.2 \mathrm{~cm} \times 50.0 \mathrm{~cm}$, henceforth referred to as the 'outer box' (see Fig. 1). A rigid lid was suspended horizontally, $2.0 \mathrm{~cm}$ below the top of the outer box, which was filled, to the height of the lid's underside, with a salt water solution of uniform density $\rho=1.028 \mathrm{~g} / \mathrm{cm}^{3}$ (i.e. the total water depth (a)

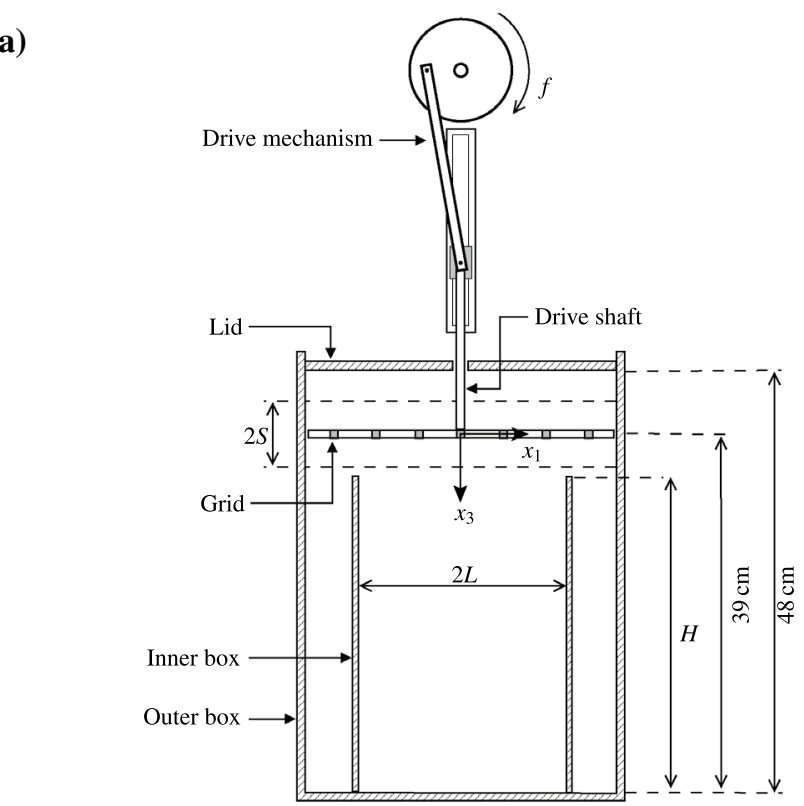

(b)

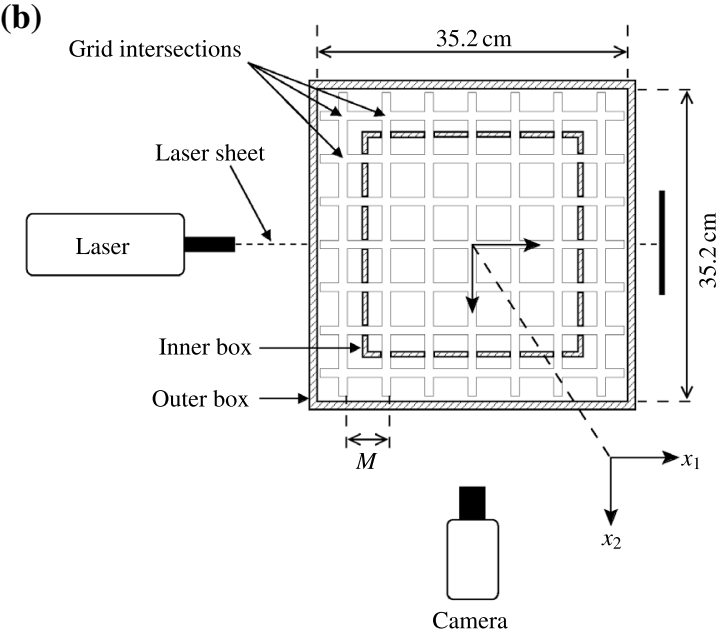

Fig. 1 Sketches showing the key components of the experimental setup. a A side view showing the positioning of the reciprocating drive mechanism, the horizontal grid and the inner and outer boxes. b A plan view showing the position of the inner box relative to the grid's mesh, and the position of the camera relative to the vertical light sheet produced by the laser. Also shown are the coordinate directions $\left(x_{1}, x_{2}, x_{3}\right)$. The set-up shown corresponds to the experiments labelled ' $\mathrm{B}$ ' in Table 1

was $48.0 \mathrm{~cm}$ ). The salt used was $\mathrm{NaCl}$. We note here that the salt water solution was required to make the seeding particles used for the PIV technique neutrally buoyant (the PIV technique is discussed in more detail in Sect. 2.2).

A planar grid was attached to the base of a drive shaft (of $1 \mathrm{~cm}$ diameter) and suspended inside the outer box with the plane of the grid horizontal (see Fig. 1a). The drive shaft passed through a circular hole of $2.6 \mathrm{~cm}$ diameter in the centre of the lid. With the grid in its neutral position (i.e., the position shown in Fig. 1a) its mid-plane was a height $39.0 \mathrm{~cm}$ above the base of the outer tank. The vertical 
oscillatory motion of the shaft and grid was driven using a cam and linear bearing to convert the rotary motion of a motorised flywheel to reciprocating vertical motion of the drive shaft, as shown in Fig. 1a. The grid's oscillation frequency $(f)$ was controlled using a potentiometer and a single fixed stroke of $S=2.5 \mathrm{~cm}$ was used throughout (here, the stroke is defined as being equal to the amplitude of the grid's motion). The grid was fabricated from stainless steel bars (of square cross-section, $1.0 \mathrm{~cm} \times 1.0 \mathrm{~cm}$ ) joined together to form a $7 \times 7$ regular array with a uniform mesh spacing $M=5.0 \mathrm{~cm}$. The corresponding solidity of the grid was $36.4 \%$. Figure $1 \mathrm{~b}$ shows a plan view of the grid. The edge conditions were chosen so that the tank sidewalls were planes of symmetry, although a narrow clearance of approximately $0.3 \mathrm{~cm}$ was required between the grid's perimeter and the internal sidewalls of the outer box. It is convenient here to define terminology that will be used throughout to denote key features of the grid: we use 'grid intersections' to denote where grid elements cross (as illustrated in Fig. 1b) and 'mid-points' as being those points on the grid that are equidistant between two adjacent grid intersections.

During initial tests to determine the effect the installation of an inner box has on the turbulence and mean-flow characteristics, consideration was given to the size and positioning of the inner box. It was found that a significant reduction in the magnitude of the mean-flow components could be obtained using a cuboid, open-ended inner box, fixed to the base of the outer box and positioned centrally with the vertical sidewalls of the inner box equidistant between the outermost and second-outermost grid intersections (as shown in Fig. 1b), and with its top edge $1 \mathrm{~cm}$ below the bottom of the grid's oscillation (as shown in Fig. 1a). For this case, therefore, the inner box had height $H=35.0 \mathrm{~cm}$ with a square internal cross-section $2 L \times 2 L$, with $2 L=24.5 \mathrm{~cm}$ (see Fig. 1a). For simplicity we henceforth refer to this as the 'optimal' case, although we stress that no detailed optimisation analysis was performed.

For conciseness, we focus here on reporting results obtained for this 'optimal' case, which will be compared with corresponding results obtained using the standard OGT set-up (with no inner box installed). However, we recall that Drayton (1993) proposed that the inner box should be positioned with its top edge a distance at least $2 M$ below the bottom of the grid's oscillation. However, our experiments showed that this was not the case, and that using an inner box of height $H=26.5 \mathrm{~cm}$ (with the same cross-section stated above), with its top edge located $2 M=10 \mathrm{~cm}$ below the bottom of the grid's oscillation, had little effect in reducing the magnitude of the mean-flow components; a comparison with this case is shown in McCorquodale (2017) [available, upon request, from the current authors].

Table 1 summarises the experimental conditions reported in this paper. The range of $f$ used is typical of that used in most previous studies (McDougall 1979; Brumley and
Table 1 A summary of the experimental conditions reported in this paper, where $M$ denotes mesh size, $S$ is the stroke, $f$ the oscillation frequency, $H$ the inner box height ('-' denotes when no inner box was installed) and the grid Reynolds number $R e$

\begin{tabular}{llllll}
\hline Experiment & $M(\mathrm{~cm})$ & $S(\mathrm{~cm})$ & $f(\mathrm{~Hz})$ & $H(\mathrm{~cm})$ & $R e$ \\
\hline $\mathrm{A} 1 \dagger$ & 5.0 & 2.5 & 1.6 & - & 2020 \\
$\mathrm{~A} 2 \dagger$ & 5.0 & 2.5 & 3.2 & - & 4050 \\
$\mathrm{~A} 3$ & 5.0 & 2.5 & 4.9 & - & 6070 \\
$\mathrm{~B} 1 \dagger$ & 5.0 & 2.5 & 1.6 & 35.0 & 2020 \\
$\mathrm{~B} 2 \dagger$ & 5.0 & 2.5 & 3.2 & 35.0 & 4050 \\
$\mathrm{~B} 3$ & 5.0 & 2.5 & 4.9 & 35.0 & 6070 \\
\hline
\end{tabular}

$\dagger$ Experiments marked were repeated to acquire PIV measurements in different regions of the flow(see Sect. 2.2 for details)

Jirka 1987; Kit et al. 1995, 1997; McCorquodale and Munro 2017) and is below the maximum recommended frequency of $7 \mathrm{~Hz}$ (McDougall 1979). In Table 1, the experiments labelled 'A' correspond to the standard OGT set-up, with no inner box installed; those labelled ' $\mathrm{B}$ ' correspond to the 'optimal' case described above (using an inner box of height $H=35.0 \mathrm{~cm}$ ). Also shown in Table 1 are values of the grid Reynolds number, defined here as

$R e \equiv M S f / v$,

where $v$ denotes kinematic viscosity. The three values considered, i.e., $R e=2020,4050$ and 6070, are representative of those used in many previous studies (Brumley and Jirka 1987; Kit et al. 1995, 1997; Nokes 1988), which are typically between $10^{3}$ and $10^{4}$.

\subsection{Velocity measurements}

Measurements of instantaneous fluid velocities were obtained using two-dimensional PIV, applied to the vertical plane through the centre of the grid (see Fig. 1b). A thin light sheet, produced by a $100 \mathrm{~Hz}$ pulsed laser (Dantec Dynamics NANO L Power 50-100), was used to illuminate small, neutrally buoyant seeding particles suspended within the water column, which act as a passive tracer as they are advected by the grid-stirred turbulence. The particles used were Pliolite with diameter range $75-125 \mu \mathrm{m}$, which when suspended within the water column had a volume fraction of $O\left(10^{-4}\right)$. The motion of the illuminated particles was recorded using a high-speed digital camera positioned to point horizontally into the tank's interior and aligned perpendicular to the plane of the vertical light sheet (see Fig. 1b). The camera was synchronised with the pulse of the laser, so the images were recorded at 100 frames per second (at $1280 \times 1024$ pixel resolution). In all experiments, images were recorded for a period of at least $240 \mathrm{~s}$. Measurements were made only after the grid had been allowed to oscillate (at a fixed frequency and stroke) for at least $15 \mathrm{~min}$ to 
ensure the turbulence within the TDR was well-established in a statistically stationary condition. PIV calculations were performed in Digiflow (Dalziel 2006), using square interrogation windows of $15 \times 15$ pixels, overlapped to achieve 10 pixel spacing between velocity vectors, which corresponded to a physical spacing between velocity vectors of approximately $0.21 \mathrm{~cm}$ to $0.28 \mathrm{~cm}$.

The velocity data were calculated and analysed relative to the right-handed coordinate system $\left(x_{1}, x_{2}, x_{3}\right)$, where $x_{3}$ denotes vertical depth below the mid-height of the grid's oscillation and $\left(x_{1}, x_{2}\right)$ are the horizontal coordinates relative to the center of the grid (see Fig. 1). The corresponding velocity components are denoted $\left(u_{1}, u_{2}, u_{3}\right)$; the two components measured using the PIV set-up described above are $u_{1}\left(x_{1}, x_{3}, t\right)$ and $u_{3}\left(x_{1}, x_{3}, t\right)$, in the central plane at $x_{2}=0$. For each experiment listed in Table 1, measurements were obtained with the camera focussed on the region $M \lesssim x_{3} \lesssim 4.5 M$ (i.e., focussing on the TDR) and in this case the instantaneous velocity data were analysed using the conventional Reynolds decomposition $u_{i}=\bar{u}_{i}+u_{i}^{\prime}$, where $u_{i}^{\prime}(\mathbf{x}, t)$ denote the fluctuating components and $\bar{u}_{i}(\mathbf{x})$ the timeaveraged mean components (the overbar notation is used throughout to denote time averaging). Previous PIV studies of OGT have typically analysed time-averaged statistical quantities using an averaging period of between $100 \mathrm{~s}$ and 120 s (Cheng and Law 2001; Lucas et al. 2016; Wan Mohtar and Munro 2013; Al-Homoud and Hondzo 2007). Here, however, an averaging period of $240 \mathrm{~s}$ was used to ensure well-converged mean and rms velocity measurements; representative data showing the convergence of time-averaged mean velocity measurements, as a function of the averaging time used, is shown in Fig. 2a.

We note that within the TDR (i.e., for $x_{3} \gtrsim 2.5 M$ ) the Kolmogorov length scale $\eta$ was estimated to lie in the range $0.03 \mathrm{~cm} \lesssim \eta \lesssim 0.13 \mathrm{~cm}$, which is finer than the physical spacing between velocity vectors computed by the PIV calculations (approximately $0.21 \mathrm{~cm}$ to $0.28 \mathrm{~cm}$ ). [The Kolmogorov length scale was estimated using the relation $\eta=v^{3 / 4} \varepsilon^{-1 / 4}$ under the assumption that, in OGT, $\varepsilon \approx 0.75\left(u_{1}^{\prime 2}\right)^{3 / 2} / \bar{\ell}$ for $x_{3} \gtrsim 2.5 M$ (Kit et al. 1997)]. Thus, the resolution used for PIV calculations was coarser than the smallest turbulent scales within the flow, such that velocity averaging occurred across interrogation windows and some turbulent fluctuations were unresolved. Consequently, the full energy content of the turbulent flow was not determined by the analysis. However, in the context of the size of the integral scales of the turbulent flow (which are of the order $2 \mathrm{~cm}$ in size, see Sect. 3.4) the range in scales of turbulent fluctuations that were under-resolved in the current analysis was small and was assumed to have little effect on the results presented in Sect. 3 since here we are primarily concerned with the large-scale structure of the flow.

A subset of the experiments listed in Table 1 (marked $\dagger$ ) were repeated (under nominally identical conditions)
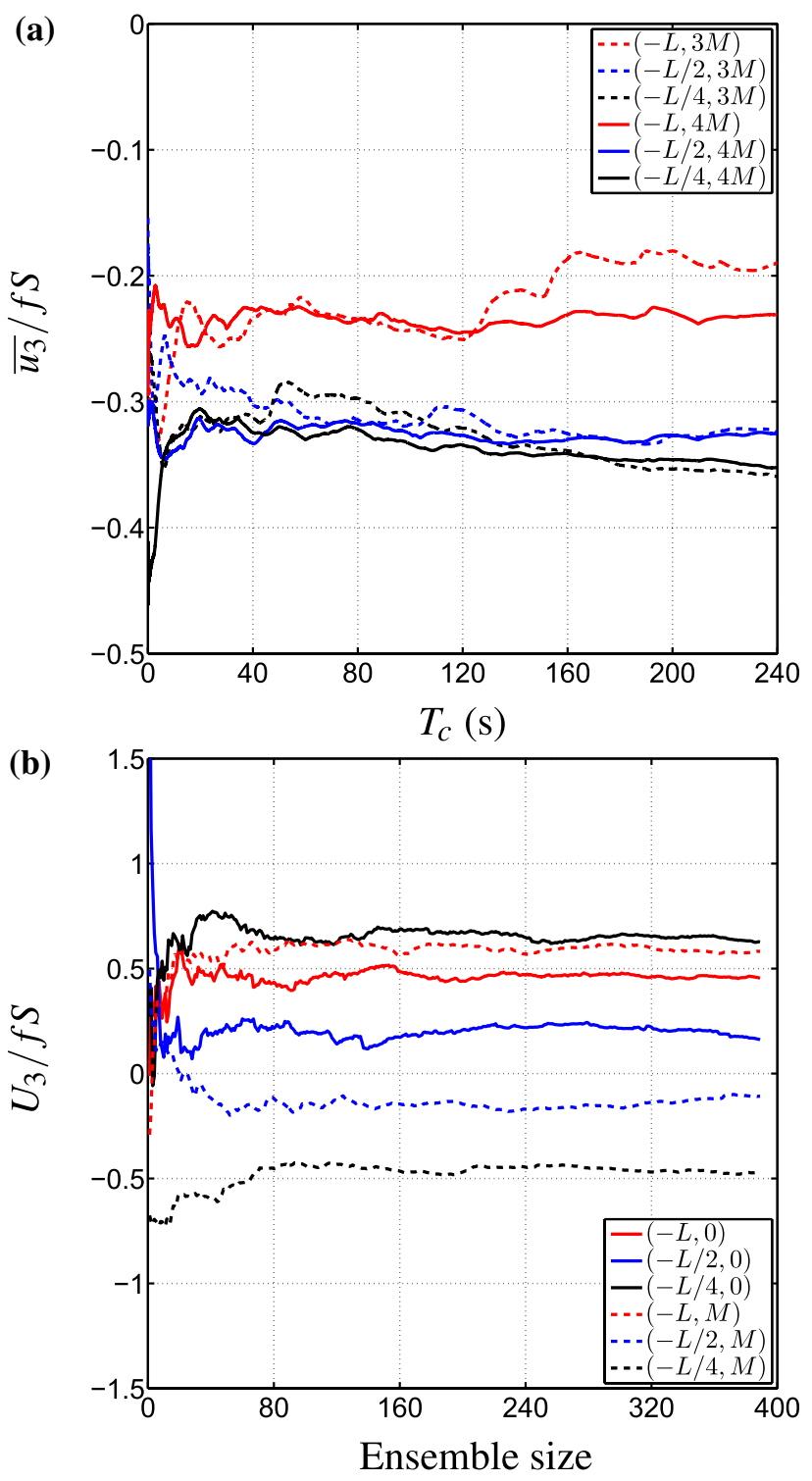

Fig. 2 a Convergence of the time-averaged vertical velocity component $\overline{u_{3}} / f S$, plotted against the averaging time used $T_{c}$. b Convergence of the ensemble-averaged vertical velocity component $U_{3} / f S$, plotted against the ensemble size used. In each panel data for six representative points from experiment $\mathrm{A} 1$ are shown [the coordinates of the points shown, on the $\left(x_{1}, x_{3}\right)$ plane, are given in the legends]; we stress that these data are also representative of the other cases reported here

to obtain PIV measurements focussed on the NGR, which were used to analyse the formation of the jets produced by the oscillating grid and, in particular, the effect the installation of the inner box had on the structure of these jets. This required analysis of the jet structures at different stages during the grid's oscillation and so time averaging was not appropriate, in this case. Instead, the near-grid-region data were analysed by ensemble averaging measurements of the fluid velocity fields taken at specific stages of the grid's 
oscillation. The ensemble-averaged mean velocity components, henceforth denoted as $U_{1}$ and $U_{3}$, were computed at eight stages of the grid's oscillation, which corresponded to when the grid was located at the points $x_{3, k}=-S \cos \left(2 \pi T_{k}\right)$, where $T_{k}=(k-1) / 8, k=1,2, \ldots, 8$. That is, $x_{3,1}$ and $x_{3,5}$ correspond, respectively, to the top and bottom of the grid's oscillation. For $R e=2020(f=1.6 \mathrm{~Hz})$ and $R e=4050$ $(f=3.2 \mathrm{~Hz})$, the available ensemble sizes were, respectively, 390 and 780, and the corresponding ensemble-averaged quantities were found to be well-converged using these ensemble sizes; representative data showing the convergence of the ensemble-averaged mean velocity measurements, as a function of the ensemble size used, is shown in Fig. 2b. We also note that for the case when no inner box was installed the data below the grid at a given $x_{3, k}$ provides a mirror image of that we would observe above the grid for $x_{3, k+4}$ (i.e., when the direction of the grid's motion is reversed). That is, here we discuss the flow observed beneath the grid and assume the flow above the grid can be inferred using the symmetry of the apparatus.

\section{Results}

\subsection{The mean-flow structure in the near-grid region with no inner box installed}

We start by describing the structure of the mean flow for the case when no inner box was installed, and by first focussing attention on the formation of jets in the NGR (which, recall, is the region $\left.\left|x_{3}\right| \lesssim 2.5 M\right)$. For this we use measurements of the ensemble-averaged mean velocity components $\left(U_{1}, U_{3}\right)$, calculated at the eight grid locations $x_{3, k}$. Figure 3 shows vector plots of $U_{1} / f S$ and $U_{3} / f S$ from Experiment A1 ( $R e=2020)$, at each $x_{3, k}$, together with corresponding measurements of the ensemble-averaged mean vorticity component $\Omega_{2} / f$ (i.e., $\Omega_{2}$ was calculated by ensemble averaging measurements of $\omega_{2}=\partial u_{1} / \partial x_{3}-\partial u_{3} / \partial x_{1}$ at each $x_{3, k}$ ) (see caption for details). We note that there are regions immediately below the grid where data have not been included; very close to the grid, where the magnitude of the fluid velocity was greatest, the PIV analysis mostly returned spurious velocity vectors, as a result of poor local correlation between interrogation windows in successive images, and so these data have not been included.

Ignoring for now the data adjacent to the tank sidewalls and, instead, focussing attention on the central region $\left|x_{1} / L\right| \lesssim 0.6$, we see that the structure of the flow is largely consistent with previous descriptions of OGT (see, for example, Thompson and Turner 1975). That is, below the lowest point of the grid's oscillation (i.e. ,below $\left.x_{3} / M=S / M=0.5\right)$ the mean-flow structure consists of a regular array of 'jets' produced by the elements of the oscillating grid, with each jet having comparable strength and consisting of a downward-directed outflow directly below grid intersections, with a corresponding upwarddirected return flow induced in the regions below the mesh voids. The jets spread, merge and (spatially) decay in the direction normal to and away from the grid, being all but fully decayed by the edge of the NGR. We stress here that the flow structure described above (and below) is of course not two-dimensional, but three-dimensional; that is, due to the symmetry of the grid (and container) we expect there to be a similar structure to the flow in the perpendicular $x_{2}$ direction.

The ensemble-averaged fields also show the presence of a regular array of counter-rotating vortex pairs that form periodically in the immediate wake of the grid, i.e., in the region $\left|x_{3} / M\right| \lesssim S / M=0.5$. That is, as the grid oscillates up and down, intense vortices are shed periodically from grid elements which then form in the immediate wake (above or below) the grid. The sense of circulation within the wake vortices depends upon the grid's direction of the motionthe vorticity within the wake vortices changes sign when the grid's direction changes. In contrast, the sense of circulation within the jet vortices remains constant with time, irrespective of the grid's direction of the motion. The vortices produced in the wake of the grid are subsequently ejected away from the grid within the vertical jets below the wake region.

We now consider the structure of the ensemble-averaged mean flow in the regions adjacent to the tank sidewalls, for $\left|x_{1} / L\right| \gtrsim 0.6$. The data in Fig. 3 show that beyond the immediate wake of the grid, for $\left|x_{3} / M\right|>S / M=0.5$, a single jet has formed below the two outermost grid intersections, on each side of the grid. These 'outer jets' exhibit significantly larger velocities and are approximately twice the size of the jet structures that form below the centre of the grid; that is, the outer and central jets have characteristic widths of order $2 M$ and $M$, respectively. As a result, the stronger outer jets penetrate to a greater depth below the grid before decaying. We also observe that the axis of the outer jets, identified by the strong vertical downward flow, is not coincident with a grid intersection, as is the case for the central jets, but instead is coincident with the midpoint between the two outermost intersections (for example, see Fig. 3e).

The outer jets form as a result of the no-slip and nopenetration conditions at the vertical sidewalls, together with the edge conditions of the grid, combining to give rise to a complicated three-dimensional flow structure in the wake (above or below) the perimeter region of the grid. At this point, we stress the inherent limitations in the twodimensional vertical-plane velocity data reported here; the data does not, of course, fully resolve the features of the three-dimensional flow produced around the perimeter of the grid. However, the two-dimensional velocity data does enable us to show the key structures that result, due 


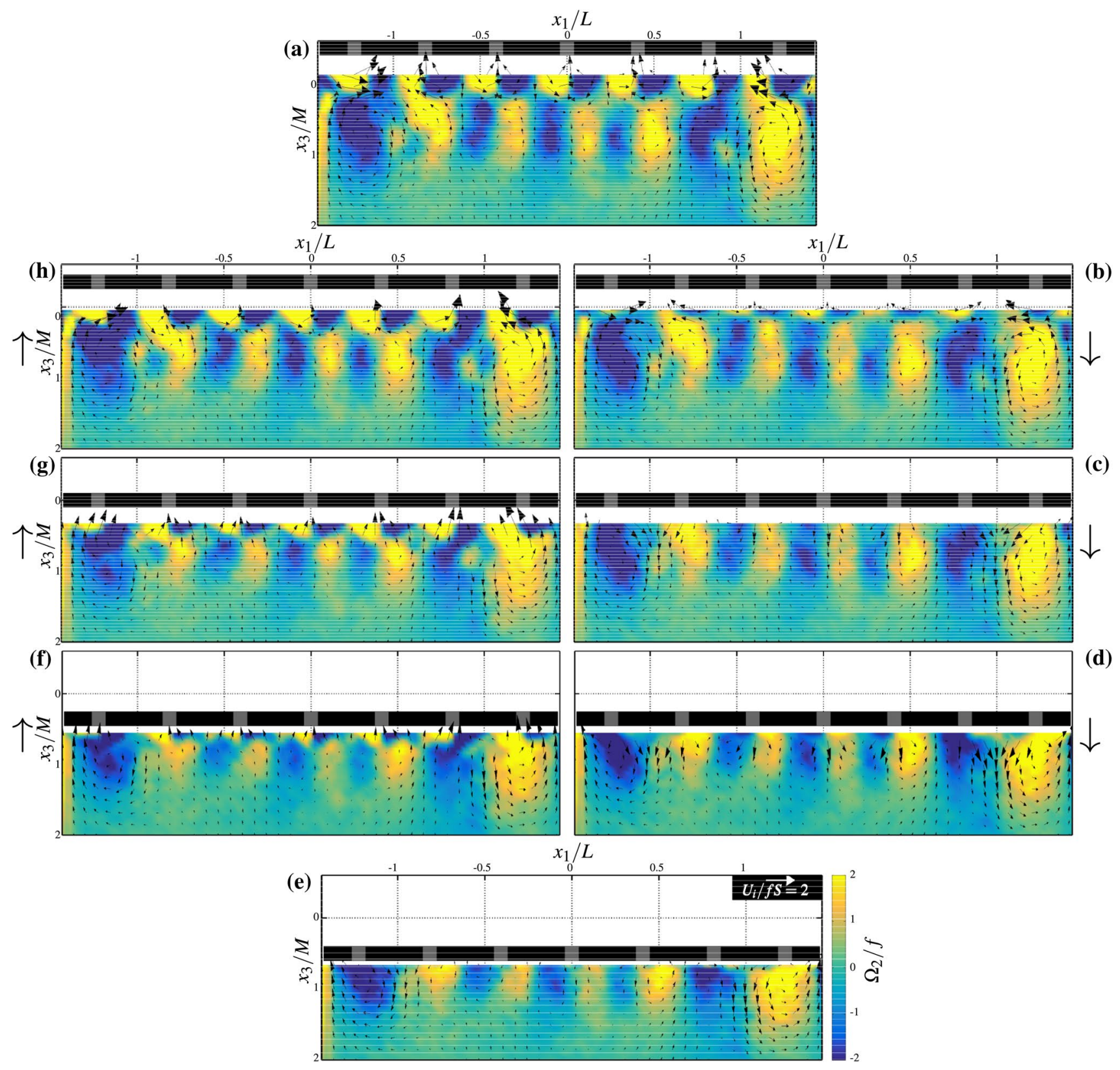

Fig. 3 Experiment A1 $(R e=2020, f=1.6 \mathrm{~Hz})$. Vector plots showing the ensemble-averaged mean velocity components $U_{1} / f S$ and $U_{3} / f S$, together with the corresponding ensemble-averaged mean vorticity $\Omega_{2} / f$, at each of the eight grid locations $x_{3, k}$ : a $x_{3,1}=-S$, b $x_{3,2}=-0.71 S$, c $x_{3,3}=0, \mathbf{d} x_{3,4}=0.71 S$, e $x_{3,5}=S$, f $x_{3,6}=0.71 S$, g $x_{3,7}=0$, h $x_{3,8}=-0.71 S$. The same colour scale for vorticity has been used in each plot, which is shown in $\mathbf{e}$, together with a reference

to this three-dimensional flow, within the NGR. That is, the data do show that as the grid ascends (Fig. 3e-h), we observe an asymmetry in the counter-rotating vortex pair that forms in the wake below the outermost grid intersection (see Fig. 3a-e). The result is that instead of observing a strong vertical flow in the wake vortices towards the grid intersection, as is the case in the central region (see velocity scale. To prevent an oversaturation of data, only every third velocity vector has been included. The instantaneous position of the grid is also shown in each plot (thick black line) and the direction of the grid's motion is shown by the arrow at the side of each plot (b-d, $\mathbf{f}-\mathbf{h})$. Note that the grid mesh spacing $M$ is shown on the $x_{1}$ axis by the spacing between neighbouring grid intersections (grey squares)

Fig. 3a), below the outermost intersection we observed a strong flow that is inclined towards the inside of the intersection, which can be seen in Fig. 3a in the wake region at $x_{1} / L \approx \pm 1.17$. We believe that this edge effect, combined with other three-dimensional edge effects and the viscous and no-penetration conditions at the sidewalls results in the merging of the jets below the two outermost grid 
elements (in the sidewall-normal direction), and ultimately in the formation of the single outer jet in this region.

The formation of the stronger outer jets has significant effects on the structure of the flow. In comparison to the central jets, the outer jets penetrate to a greater depth below the grid before decaying. This greater penetration of outer jets has previously been proposed to induce large-scale circulations that affect the entire domain (Drayton 1993). The data reported here are consistent with this proposal, as illustrated in Fig. 4 which shows a vector plot of the time-averaged mean velocity components $\overline{u_{1}} / f S$ and $\overline{u_{3}} / f S$ for Experiment A1 $(R e=2020)$, together with corresponding measurements of the time-averaged mean vorticity component $\overline{\omega_{2}}$ (note the different velocity vector scale used in Fig. 4 relative to Fig. 3). These data show that, in the absence of an inner box there is a significant (on this plane predominantly upward) time-averaged mean flow induced in both the near-grid and turbulent-diffusive regions. For reference we note that the average values of mean-flow intensity in the turbulentdiffusive region, computed as a spatial average across the region given by $2.5 \leq x_{3} / M \leq 4.5$ and $\left|x_{1} / L\right| \leq 0.7$, are

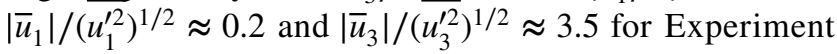
A1; these values are also representative for the corresponding data obtained in Experiments A2 and A3. Time-averaged mean flow fields indicating the presence of large-scale circulations, similar to the data shown in Fig. 4, have also been reported in a range of previous OGT studies (see, for example, Bennett et al. 2014; McKenna and McGillis 2004; Dohan and Sutherland 2002).

\subsection{The mean-flow structure in the near-grid region with inner box installed}

For comparison, Fig. 5 shows the ensemble-averaged data from Experiment B1 $(R e=2020)$, with the inner box installed in the 'optimal' position; for reference, the position of the inner box sidewalls are shown by the vertical black lines. The same scales for velocity and vorticity have been used in Figs. 3 and 5 to facilitate a direct comparison. Note that the data for regions immediately below the grid, where the PIV algorithm produced spurious velocity vectors, has not been included. In addition, data immediately outside the inner box sidewalls has not been included in Fig. 5 as a result of substantially reduced image quality in these regions, due to the reflection and refraction of light from the inner box sidewalls, over which the PIV analysis also failed.

The data in Fig. 5 shows that the presence of the inner box has little, if any, affect on the vortices that form in the immediate wake of the grid, where we again observe a regular array of vortex pairs forming periodically below (or above) each intersection, with the same edge effects described above again evident below (or above) the two outermost grid intersections. That is, the mechanisms responsible for the formation of the merged outer jets are still present. However, Fig. 5 shows that below the grid's immediate wake, the presence of the inner box sidewalls at $\left|x_{1} / L\right|=1$ blocks the formation of the merged outer jets, instead resulting in largescale recirculations forming in the compartment between the inner and outer boxes, which extend up to and beyond $x_{3} \approx 2 M$. (We no further consider the compartment region between the inner and outer containers and henceforth focus attention on the flow conditions within the interior of the inner box.) Notably, similar large-scale recirculations are not induced inside the inner box. Instead, the array of jet structures that form across the interior of the inner box are of a more uniform strength and structure. The regular structure of these jets is attributed to how the merged outer jets are blocked by the inner box sidewalls, which gives rise to flow separation at the top rim of the inner box, resulting in the formation of the jets inclined towards the centre of the
Fig. 4 Experiment A1 $(R e=2020, f=1.6 \mathrm{~Hz})$. Vector plots showing the time-averaged mean velocity components $\bar{u}_{1} / f S$ and $\bar{u}_{3} / f S$, together with the corresponding time-averaged mean vorticity component $\bar{\omega}_{2} / f$. A reference velocity scale and vorticity scale is shown. To prevent oversaturation, only every third velocity vector has been included. For reference the grid is shown at the lowest point within its oscillation

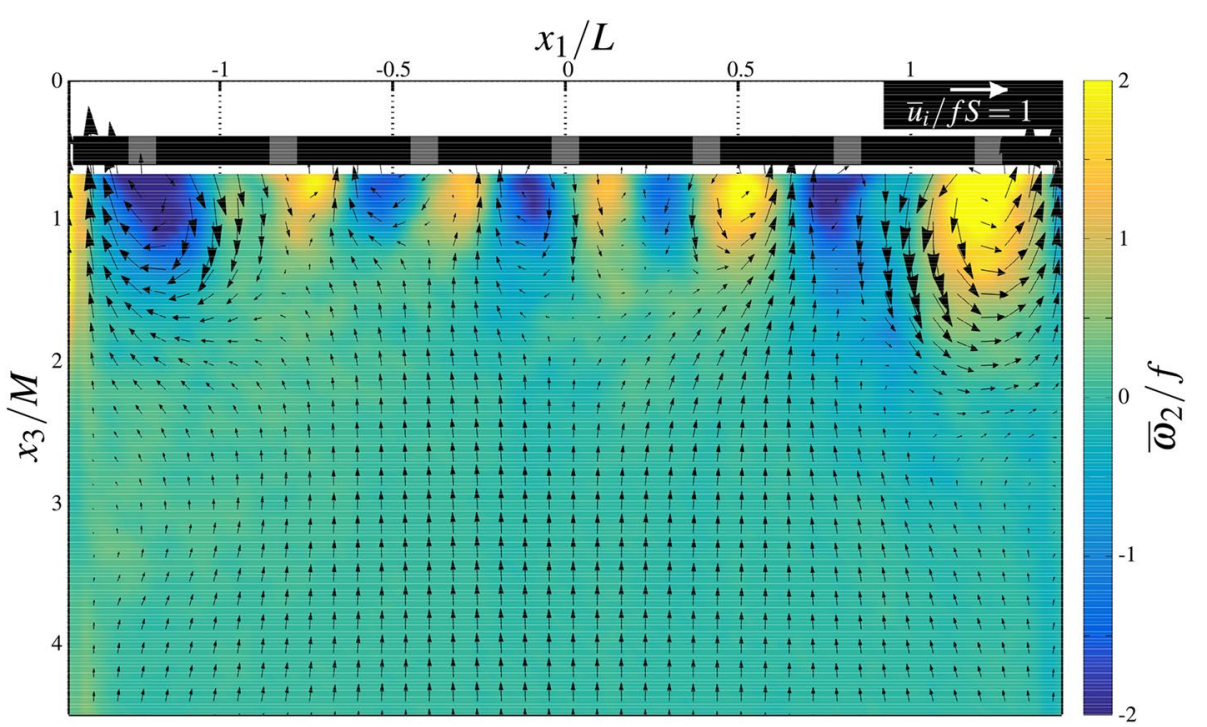




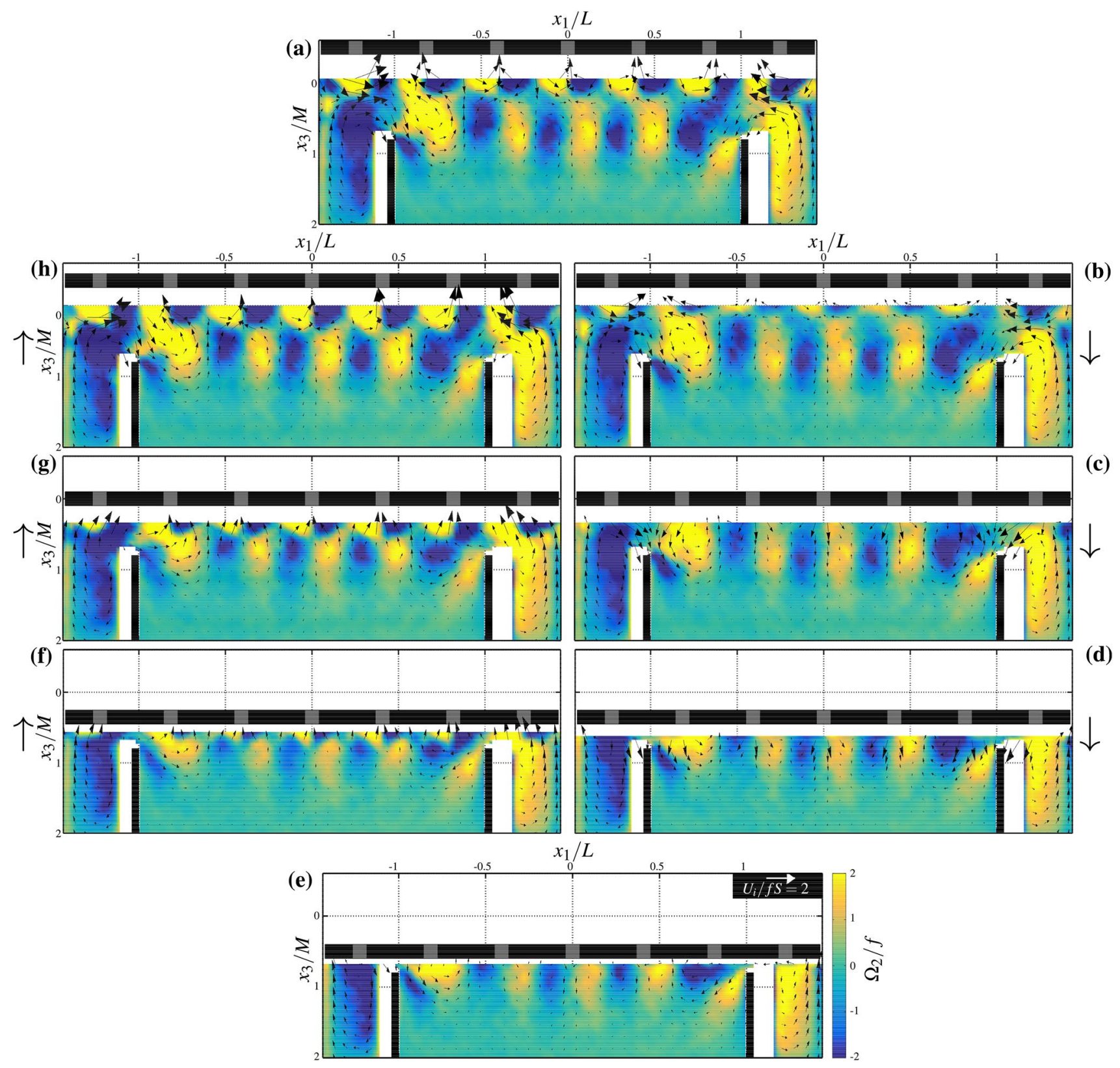

Fig. 5 Experiment $\mathrm{B} 1(R e=2020, f=1.6 \mathrm{~Hz})$. Vector plots showing the ensemble-averaged mean velocity components $U_{1} / f S$ and $U_{3} / f S$, together with the corresponding ensemble-averaged mean vorticity $\Omega_{2} / f$, at each of the eight grid locations $x_{3, k}$ : a $x_{3,1}=-S$, b $x_{3,2}=-0.71 S$, c $x_{3,3}=0, \mathbf{d} x_{3,4}=0.71 S$, e $x_{3,5}=S, \mathbf{f} x_{3,6}=0.71 S$, g $x_{3,7}=0$, h $x_{3,8}=-0.71 S$. The same colour scale for vorticity has

grid. Both the inclined and central jets spread, merge and (spatially) decay in the direction normal to and away from the grid, being all but fully decayed by the edge of the NGR.

The results of the time-averaged mean flow, shown in Fig. 6, indicate that within the inner box a (non-uniform) mean flow is evident within the TDR, but is much weaker in comparison to the mean flow observed when no inner box is installed (see Fig. 4). Note that, so that direct been used in each plot, which is shown in (e), together with a reference velocity scale. To prevent an oversaturation of data, only every third velocity vector has been included. In (b-d) and (f-h) the direction of the grid's motion is shown by the arrow at the side of each plot

comparison can be made, the scales for velocity and vorticity in Fig. 6 are the same as in Fig. 4. For reference we note that the average values of mean-flow intensity in the TDR, computed as a spatial average across the region given by $2.5 \leq x_{3} / M \leq 4.5$ and $\left|x_{1} / L\right| \lesssim 0.5$, are $\left|\bar{u}_{1}\right| /\left(\overline{u_{1}^{\prime 2}}\right)^{1 / 2} \approx 0.3$ and $\left|\bar{u}_{3}\right| /\left(\overline{u_{3}^{\prime 2}}\right)^{1 / 2} \approx 0.6$ for Experiment B1 (which are also representative of Experiments B2-B3); comparing with the corresponding values obtained for Experiment A1 
Fig. 6 Experiment $\mathrm{B} 1$ $(R e=2020, f=1.6 \mathrm{~Hz})$. Vector plots showing the time-averaged mean velocity components $\bar{u}_{1} / f S$ and $\bar{u}_{3} / f S$, together with the corresponding time-averaged mean vorticity component $\bar{\omega}_{2} / f$. A reference velocity scale and vorticity scale is shown. To prevent oversaturation only every third velocity vector has been included. For reference the grid is shown at the lowest point within its oscillation

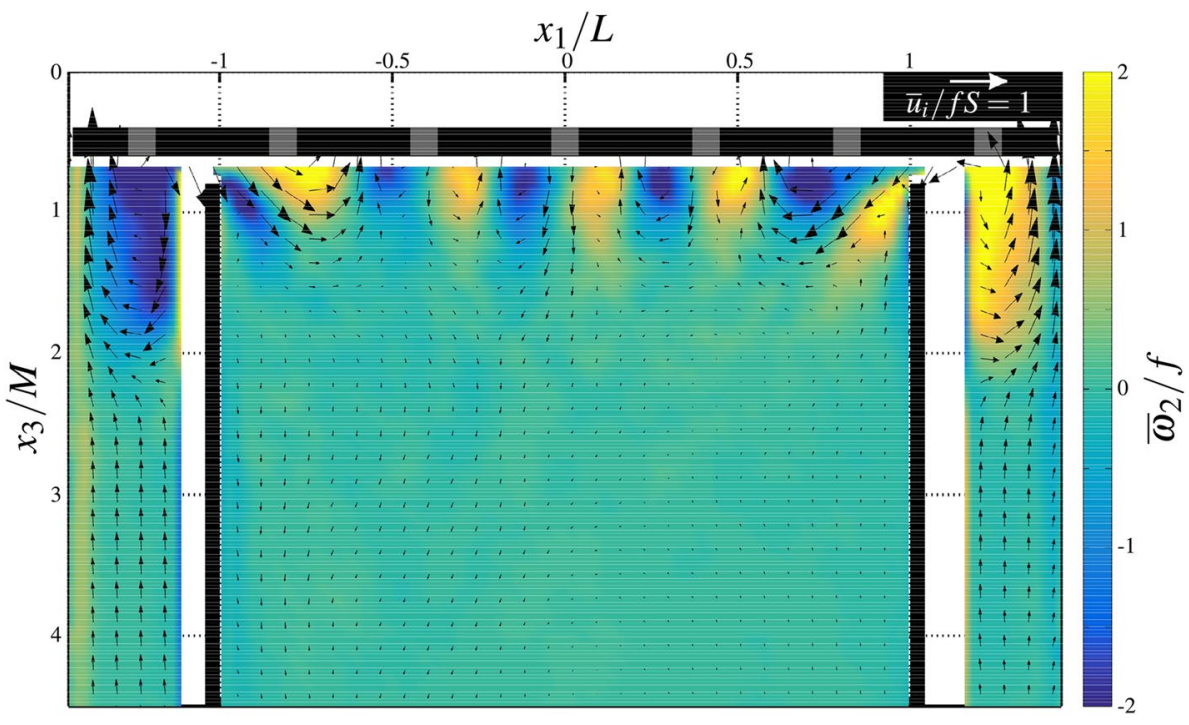

$\left(\left|\bar{u}_{1}\right| /\left(\overline{u_{1}^{\prime 2}}\right)^{1 / 2} \approx 0.2\right.$ and $\left.\left|\bar{u}_{3}\right| /\left(\overline{u_{3}^{\prime 2}}\right)^{1 / 2} \approx 3.5\right)$ shows that the installation of the inner box results in a significant reduction in the magnitude of the (predominantly vertical) mean flow. In addition, we stress that the structure of the mean flow described above (i.e., as observed in the central vertical plane $x_{2}=0$ ) is consistent with observations made during preliminary experiments in which the light sheet was used to illuminate seeding particles within the flow in a number of additional vertical planes across the width of the tank (i.e., for $x_{2} \neq 0$ ). That is, these preliminary experiments indicated that, within the inner box, the TDR was free from large-scale circulations. These results are consistent with the proposal of Drayton (1993) that mean flow arises when there is a significant difference in the relative strength of the jets produced by the oscillating grid in different regions of the tank; with the inner box installed, the jets are of approximately equal strength and penetrate to approximately equal depth before breaking down.

\subsection{Effect of mean flow on turbulent structure}

We now describe how the installation of the inner box affects the spatial structure of the turbulence, in both the NGR and TDR. We note that in previous studies the qualitative characteristics of the turbulence produced by an oscillating grid were not found to exhibit Reynolds number effects across the fairly limited Reynolds number ranges used (typically between $10^{3}$ and $10^{4}$ ) (see, for example, Hopfinger and Toly 1976; Wan Mohtar and Munro 2013). Likewise, the qualitative characteristics of the experimental data reported here were also found to be little different over the range of Reynolds numbers considered $\left(R_{G}=2020\right.$ to 6070), and so we present here results for the case $R e_{G}=4050$, which are representative.
First, we consider field measurements of the timeaveraged degree of isotropy. The idealised model for OGT assumes the turbulence produced is homogeneous and iso-

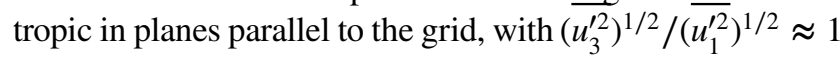
within the TDR. In line with this assumption, previous experimental studies have reported the degree of isotropy

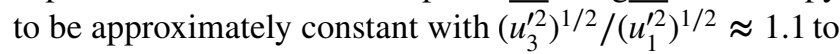
1.4 in the TDR (Hopfinger and Toly 1976; Hopfinger and Linden 1982; Kit et al. 1997). However, to our knowledge, field measurements of the (spatially non-uniform) degree of isotropy have not previously been reported in studies of OGT and the structure of the turbulence in the NGR is largely unknown. We stress that an understanding of the degree of isotropy of the turbulence is necessary to ensure the validity of subsequent statistical analysis. That is, in previous OGT studies it is common for statistical quantities to be spatially averaged (see for example Orlins and Gulliver 2003; Wan Mohtar and Munro 2013; Bennett et al. 2014) over regions thought to be approximately homogeneous and isotropic and which are not affected by the presence of the tank side walls. However, few studies have reported measurements that can justify the spatial averages used. At best, previous work has shown spatial distributions of turbulent velocity components over limited regions of the tank (Lucas et al. 2016; McDougall 1979; Cheng and Law 2001), which do not show key features of the flow that we identify here.

The colour maps in Fig. 7 show measurements of the time-averaged degree of isotropy, $\left(\overline{u_{3}^{\prime 2}}\right)^{1 / 2} /\left(\overline{u_{1}^{\prime 2}}\right)^{1 / 2}$, from Experiments A2 and B2 (see caption for details). To illustrate the effects of turbulent production due to the mean flow, in each plot the time-averaged mean velocity components $\left(\bar{u}_{1} / f S, \bar{u}_{3} / f S\right)$ have been superimposed on top of the isotropy field. Clearly, the data shown in Fig. 7 resolves only the central vertical plane inside the container; however, due to the symmetry of the grid and container we anticipate a 


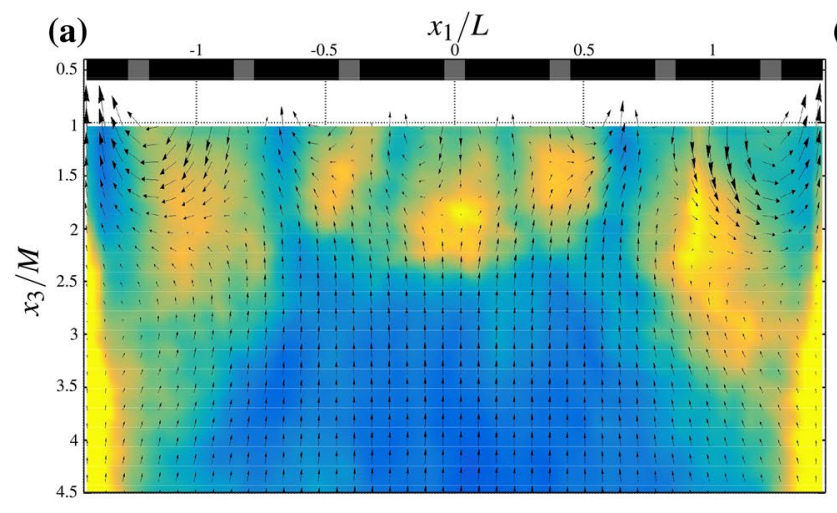

Fig. 7 Colour maps showing the degree-of-isotropy field $\left(u_{3}^{\prime 2}\right)^{1 / 2} /\left(u_{1}^{\prime 2}\right)^{1 / 2}$ together with vector plots showing the time-averaged components of mean velocity $\bar{u}_{i} / f S$. The data shown are a Experiment $\mathrm{A} 2$ and $\mathbf{b}$ Experiment B2. In each case, $R e=4050, f=3.6 \mathrm{~Hz}$. The same colour scale for isotropy has been used in each plot, which

similar structure (qualitatively) in $\left(\overline{u_{3}^{\prime 2}}\right)^{1 / 2} /\left(\overline{u_{1}^{\prime 2}}\right)^{1 / 2}$ in the perpendicular $x_{2}$-direction.

The data in Fig. 7a shows that in the NGR, with no inner box installed, the turbulence is clearly anisotropic; that is, the degree-of-isotropy field is not uniform, but instead

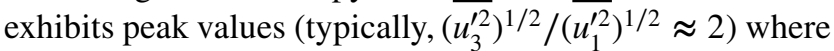
the spatial gradients in the (time-averaged) mean velocity components are greatest. Inspection of Fig. 7a shows that, in the NGR, the peak values of $\left(\overline{u_{3}^{\prime 2}}\right)^{1 / 2} /\left(\overline{\left.u_{1}^{\prime 2}\right)^{1 / 2}}\right.$ occur roughly below each grid intersection where the individual jets produced by the grid break down. This is attributed to localised anisotropic turbulent production; in these regions the spatial gradients of $\bar{u}_{3}$ exceed the spatial gradients of $\bar{u}_{1}$ such that the production of $\left(u_{3}^{\prime 2}\right)^{1 / 2}$ is greater than the production of $\left(\overline{u_{1}^{\prime 2}}\right)^{1 / 2}$

Within the TDR (i.e., $x_{3} \gtrsim 2.5 M$ ), where the jet structures have broken down, the turbulence is approximately isotropic, but only over the central region $\left|x_{1} / L\right| \lesssim 0.5$; in this region the degree of isotropy is approximately uniform with $\left(u_{3}^{\prime 2}\right)^{1 / 2} /\left(\overline{\left.u_{1}^{\prime 2}\right)^{1 / 2}} \approx 0.8\right.$. However, as the sidewalls are approached the turbulence becomes increasingly anisotropic, with $\left(\overline{u_{3}^{\prime 2}}\right)^{1 / 2} /\left(\overline{u_{1}^{\prime 2}}\right)^{1 / 2} \gtrsim 2$ in close proximity to the wall. The increase of $\left(u_{3}^{\prime 2}\right)^{1 / 2} /\left(u_{1}^{\prime 2}\right)^{1 / 2}$ in the sidewall region can be understood in light of previous work studying the interaction of zero-mean-shear turbulence with boundaries (Hunt and Graham 1978; Hannoun et al. 1988; McCorquodale and Munro 2017). That is, in zero-mean-shear turbulence the no-penetration boundary condition acts to inhibit only the boundary-normal turbulent fluctuations (i.e., $\left(\overline{u_{1}^{\prime 2}}\right)^{1 / 2}$ at the sidewalls) over a layer approximately equal in thickness to the integral length scale of the turbulence. It is only within the thin viscous sublayer, immediately adjacent to the boundary, that the no-slip condition also acts to inhibit the boundary-tangential turbulent fluctuations (driven by

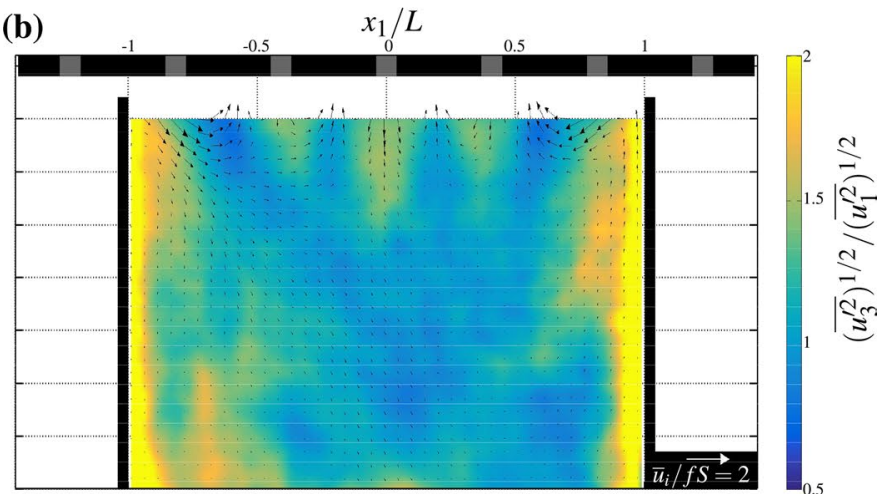

is shown in $\mathbf{b}$ together with a reference velocity scale. To prevent an oversaturation of data, only every third velocity vector has been included. For reference the grid is shown at the lowest point within its oscillation

the dissipation of TKE), thus giving rise to the increase in anisotropy observed here.

For comparison, Fig. 7b shows corresponding data obtained with the inner box installed in the 'optimal' position. Again, the turbulence is anisotropic in the NGR, although the degree of anisotropy is significantly reduced, with peak values of $\left(\overline{u_{3}^{\prime 2}}\right)^{1 / 2} /\left(\overline{\left.u_{1}^{\prime 2}\right)^{1 / 2}} \approx 1\right.$. 3 . The reduction in anisotropy is attributed to a reduction in the production

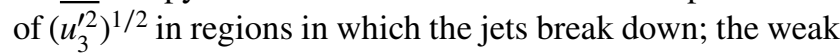
time-averaged mean flow in the turbulent-diffusive region gives rise to smaller spatial gradients in the mean flow (i.e., a reduction in the magnitude of $\partial u_{3} / \partial x_{i}$ ) over these regions relative to when no inner box is installed. Within the TDR, the turbulence over the central region, $\left|x_{1} / L\right| \lesssim 0.5$, is effec-

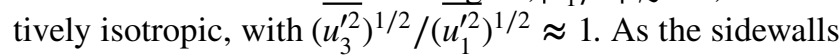
are approached, and for the same reasons given above, the turbulence again becomes increasingly anisotropic with peak values of $\left(\overline{u_{3}^{\prime 2}}\right)^{1 / 2} /\left(\overline{u_{1}^{\prime 2}}\right)^{1 / 2} \gtrsim 2$ observed close to the wall.

The effect the tank sidewalls have on the degree of isotropy can be seen more clearly in Fig. 8, which shows spatially averaged measurements of $\left(\overline{u_{3}^{\prime 2}}\right)^{1 / 2} /\left(\overline{u_{1}^{\prime 2}}\right)^{1 / 2}$ from Experiments A1-A3 and Experiments B1-B3 (see caption for details). In Fig. $8 \overline{\left(u_{3}^{\prime 2}\right)^{1 / 2}} /\left(\overline{u_{1}^{\prime 2}}\right)^{1 / 2}$ has been spatially averaged in the $x_{3}$ direction (denoted $\langle\cdot\rangle_{3}$ ) over the TDR given by $2.5 \leq x_{3} / M \leq 4.5$; in this way, the data have been spatially averaged only over those regions in which the field $\left(\overline{u_{3}^{\prime 2}}\right)^{1 / 2} /\left(\overline{\left.u_{1}^{\prime 2}\right)^{1 / 2}}\right.$ is approximately homogeneous. These data confirm that the turbulence produced by the oscillating grid is approximately isotropic only over the central region, $\left|x_{1} / \zeta\right| \lesssim 0.5$; outside of this region the anisotropy of the turbulence increases as the sidewalls are approached. We stress this is a key limitation of OGT that, to our knowledge, has not previously been reported; we first identified and reported this effect in a recent, related study (McCorquodale and Munro 2017) using two grid types (the $7 \times 7$ grid used 


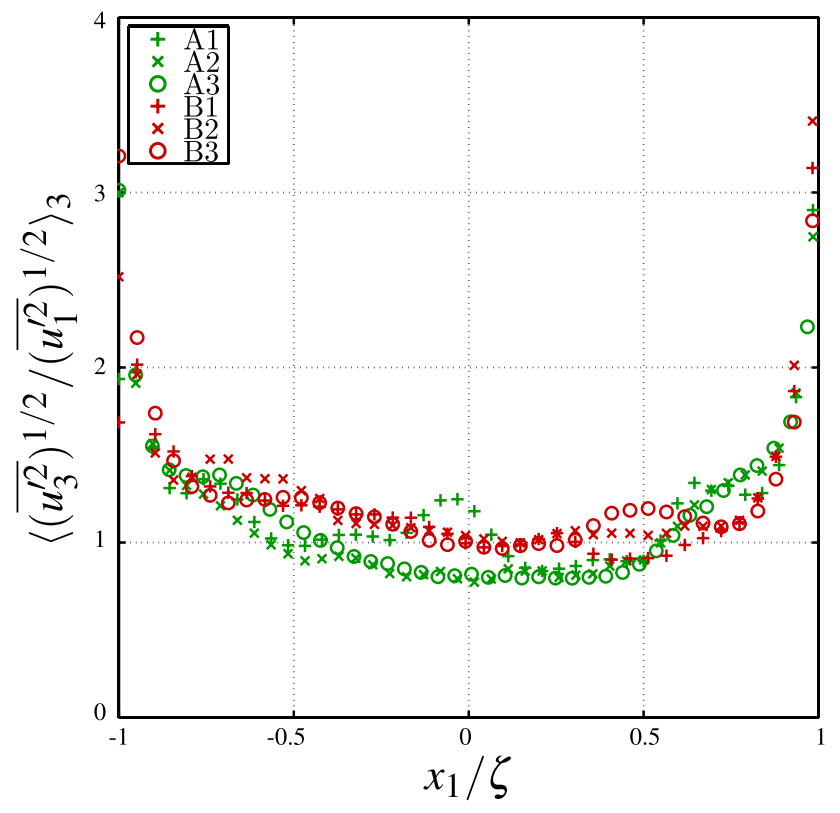

Fig. 8 Plot showing spatially averaged (in the $x_{3}$-direction) measurements of the degree of isotropy $\left\langle\left(\overline{u_{3}^{\prime 2}}\right)^{1 / 2} /\left(u_{1}^{\prime 2}\right)^{1 / 2}\right\rangle_{3}$ plotted against the horizontal $x_{1}$ coordinate which has been scaled by $\zeta$, where $\zeta$ is equal to the half-width of the outer box for Experiments A1-A3 and $\zeta=L$ for Experiments B1-B3. To prevent oversaturation, only every third data point has been plotted for each experiment. The data for all conditions are shown separately [see legend]

here, with mesh spacing $M=5 \mathrm{~cm}$, and a $9 \times 9$ grid with mesh spacing $M=3.8 \mathrm{~cm}$ ). However, note that with no inner box installed the turbulence is weakly anisotropic (i.e., $\left(\overline{u_{3}^{\prime 2}}\right)^{1 / 2} /\left(\overline{u_{1}^{\prime 2}}\right)^{1 / 2} \approx 0.8$ ) over the central region. The cause of the loss of isotropy, relative to experiments with the inner box installed, is currently unclear. Note also that here the magnitude of this data are slightly lower than in previous studies of OGT (for example, $\left(\overline{u_{3}^{\prime 2}}\right)^{1 / 2} /\left(\overline{u_{1}^{\prime 2}}\right)^{1 / 2} \approx 1.1--1.4$ in Kit et al. 1997; Hopfinger and Toly 1976; De Silva and Fernando 1994); we attribute this effect, at least in part, to the fact that here we have excluded the anisotropic sidewall

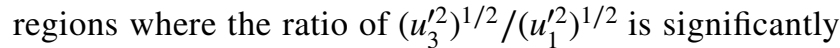
higher.

Further insight into the effect the installation of the inner box has on the turbulence can be obtained by considering measurements of the rms turbulent velocity components $\left(\overline{u_{1}^{\prime 2}}\right)^{1 / 2}$ and $\overline{\left(u_{3}^{\prime 2}\right)^{1 / 2}}$. The spatial distribution of each component exhibits the same key qualitative characteristics, so for brevity we consider here only the vertical velocity component. The colour maps in Fig. 9 show measurements of $\left(\overline{u_{3}^{\prime 2}}\right)^{1 / 2} / f S$ from experiment A2 (Fig. 9a) and experiment B2 (Fig. 9b), plotted against $x_{1} / L$ and $x_{3} / M$. To illustrate the effects of turbulent transport due to turbulent fluctuations, in each plot the time-averaged turbulent-flux components $\overline{u_{1}^{\prime} u_{3}^{\prime} u_{3}^{\prime}} /(f S)^{3}$ and $\overline{u_{3}^{\prime} u_{3}^{\prime} u_{3}^{\prime}} /(f S)^{3}$ have been superimposed on top of colour maps. We note that the data from each experiment has been divided to show separately the behaviour in the NGR (Fig. 9a,i and b,i) and TDR (Fig. 9a,ii and b,ii). To better resolve the structure of the flow different colour scales and vector scales have been used in these regions (see caption for details).

Figure 9a shows that close to the grid (and in the absence of an inner box) peak values of $\left(\overline{u_{3}^{\prime 2}}\right)^{1 / 2}$ are observed along the axes of the jets that form in this region, with maximum values attained at $x_{1} / L \approx \pm 1$, coincident with the axis of the merged outer jets. These effects can be attributed to turbulent production as the mean kinetic energy of the jets

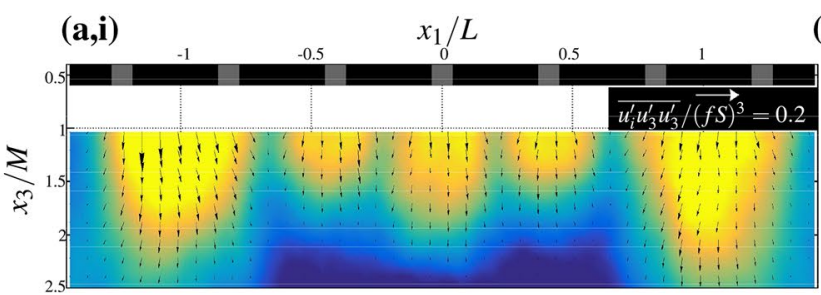

(a,ii)

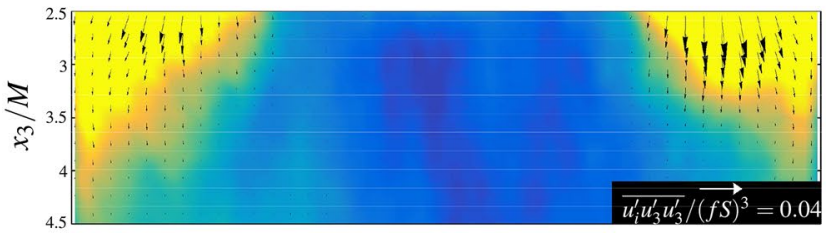

Fig. 9 Vector plots showing the time-averaged components of turbulent energy flux $\overline{u_{i}^{\prime} u_{3}^{\prime} u_{3}^{\prime}} /(f S)^{3}$ (where $i=1,3$ denote the horizontal and vertical flux, respectively), which have been superimposed on top of colour maps showing the vertical rms velocity field $\left(u_{3}^{\prime 2}\right)^{1 / 2} / f S$. The data are from a Experiment $\mathrm{A} 2$ and b Experiment B2 (with $R e=4050$ and $f=3.6 \mathrm{~Hz}$ in both cases). To provide a meaningful

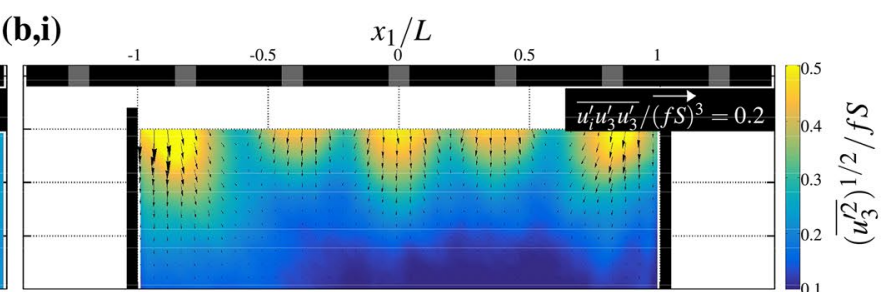

(b,ii)

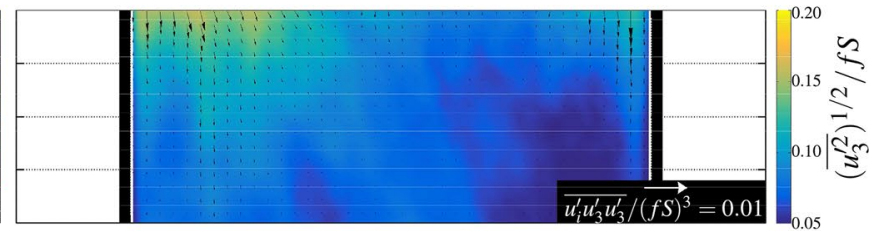

scale throughout the flow the data for each experiment has been plotted over two sub-figures. Colour scales and reference energy flux vectors are provided in each sub-figure; note the different scales used. To prevent an oversaturation of data, only every third velocity vector has been included. For reference the grid is shown at the lowest point within its oscillation 
is converted to TKE. Note, however, that since the merged outer jets are more energetic than the jets over the central region $\left(\left|x_{1} / L\right| \lesssim 0.6\right)$ there is greater production of TKE near the edge of the grid than near the centre of the grid. As a result, the turbulence produced within the NGR is strongly inhomogeneous on horizontal planes.

Further below the grid, as the jet structures spread and break down in the grid-normal direction, $\left(u_{3}^{\prime 2}\right)^{1 / 2}$ exhibits a more uniform structure over the central region of the tank $\left(\left|x_{1} / L\right| \lesssim 0.65\right)$, as the bottom of the NGR is approached. However, over this region a rapid reduction in the magnitude of $\left(\overline{u_{3}^{\prime 2}}\right)^{1 / 2}$ occurs. This is attributed to the effects of transport of turbulent energy due to the mean flow; the approximately uniform upwards-directed mean flow in the turbulence diffusive region (identified previously in Fig. 4) advects turbulence of low TKE up towards the NGR where it interacts with the (more energetic) turbulence produced by grid. Conversely, the merged outer jets do not fully breakdown within the NGR, but instead propagate (albeit diminished in strength) to $x_{3} / M \approx 4$, as shown in Fig. 9a,ii. Figure 9a,ii indicates that these outer jets are, at least in part, maintained by the transport of turbulent energy by the turbulent fluctuations. That is, the larger flux of turbulent energy close to the tank side walls than in the centre of the tank contributes to the inhomogeneity across the horizontal plane that exists in the turbulent-diffusive region. In turn, the horizontal inhomogeneity results in concentrated transport of energy close to the tank walls, preserving the structure of the outer jets.

For comparison, Fig. 9b shows corresponding data obtained with the inner box installed. In accordance with the results in Fig. 9a (Experiment A2), peak values of $\left(\overline{u_{3}^{\prime 2}}\right)^{1 / 2}$ are observed along the axes of the jets that form in the NGR. However, when the individual jets of approximately uniform strength break down, the turbulent production has a more regular structure across the entire width of the inner box. As a result, Fig. 9b indicates the turbulence produced within the NGR is more homogeneous than when the inner box was absent. In addition, the weak mean flow within the TDR gives rise to smaller production terms than for experiment A2 and, in comparison, a slight reduction in the magnitude of $\left(\overline{u_{3}^{\prime 2}}\right)^{1 / 2}$ throughout the NGR. Note, however, that the reduction in mean-flow intensity also inhibits the transport effects due to the mean flow that were identified for Experiment A2 at the edge of the NGR. As a result of the more homogeneous turbulence produced in the NGR, the coherent jet structures remain mostly confined to the NGR. That is, the turbulent energy flux is significantly reduced in magnitude close to the tank side walls, relative to experiments with no inner box installed (recall that different vector scales for energy flux are used in Figs. 9a,ii and b,ii), such that the turbulent transport is more uniform across horizontal planes. Note, however, that Fig. $9 \mathrm{~b}$ does indicate that there remains a weak preferential transport of turbulent energy close to the tank walls (relative to the center of the tank). This indicates that some intermittent jet structures may still exist close to the side walls when the inner box is installed, albeit in a much less energetic form than when no inner box is installed. Inspection of the instantaneous velocity fields confirms the presence of intermittent energetic structures in the TDR that originate from coherent jets in the NGR, although these intermittent structures are not confined to regions close to the inner box sidewalls and are also present within the central region of the tank.

\subsection{Comparison of turbulent structure with standard model of OGT}

Finally, we compare measurements of the key turbulence statistics $\left(\left(\overline{u_{1}^{\prime 2}}\right)^{1 / 2},\left(\overline{u_{3}^{\prime 2}}\right)^{1 / 2}\right.$ and $\left.\bar{\ell}\right)$ with the standard model given in Eq. (1). For the purposes of these comparison, attention is restricted to the TDR (i.e., $2.5 M \leqslant x_{3} \leqslant 4.5 M$ ), with the anisotropic near-sidewall regions excluded.

Figure 10a, b show spatially averaged measurements of $\left\langle\left(\overline{u_{1}^{\prime 2}}\right)^{1 / 2}\right\rangle_{1}$ and $\left\langle\left(\overline{u_{3}^{\prime 2}}\right)^{1 / 2}\right\rangle_{1}$, from experiments A and B, plotted against depth $x_{3}$ (see caption for details); $\langle\cdot\rangle_{1}$ is used to denote a spatial average in the $x_{1}$-direction across the central region of the container, away from the anisotropic sidewall regions (i.e., $\left|x_{1} / \zeta\right| \lesssim 0.5$, where $\zeta=17.6 \mathrm{~cm}$ for experiments A and $\zeta=L$ for experiments B). Also shown are error bars indicating uncertainty within the experimental measurements. That is, a notable source of uncertainty in OGT arises from the flow variability that occurs across experiments repeated under nominally identical conditions. In particular, McKenna and McGillis (2004) showed that the measured magnitude of mean-flow components and turbulent fluctuations vary by as much as $25 \%$ and $15 \%$, respectively, across separate experiments performed under the same conditions. Estimates of uncertainty associated with this variability, when using the current apparatus, have been determined using data previously reported by the current authors (McCorquodale and Munro 2018). That is, McCorquodale and Munro (2018) reported measurements of five ensembles of experiments repeated under nominally identical conditions. The standard deviations of the corresponding measurements of $\left\langle\left(\overline{u_{1}^{\prime 2}}\right)^{1 / 2}\right\rangle_{1}$ and $\left\langle\left(\overline{u_{3}^{\prime 2}}\right)^{1 / 2}\right\rangle_{1}$, denoted $\sigma_{u_{1}^{\prime}}$ and $\sigma_{u_{3}^{\prime}}$, computed across each ensemble were given by $\sigma_{u_{1}^{\prime}} \approx 0.06\left\langle\left(\overline{u_{1}^{\prime 2}}\right)^{1 / 2}\right\rangle_{1}$ and $\sigma_{u_{3}^{\prime}} \approx 0.06\left\langle\left(\overline{u_{3}^{\prime 2}}\right)^{1 / 2}\right\rangle_{1}$. These estimates of uncertainty are shown as the error bars in Fig. 10a, b.

The data in Fig. 10a, b show that with no inner box installed (Experiments A1-A3), Eq. (1) is a poor fit for the observed spatial decay of $\left(\overline{u_{1}^{\prime 2}}\right)^{1 / 2}$ and $\left(u_{3}^{\prime 2}\right)^{1 / 2}$. Recall, however, that Eq. (1a) is derived from the transport equation of TKE under the assumptions of zero mean flow, homogeneity (in planes parallel to the grid) and negligible viscous transport; under these 

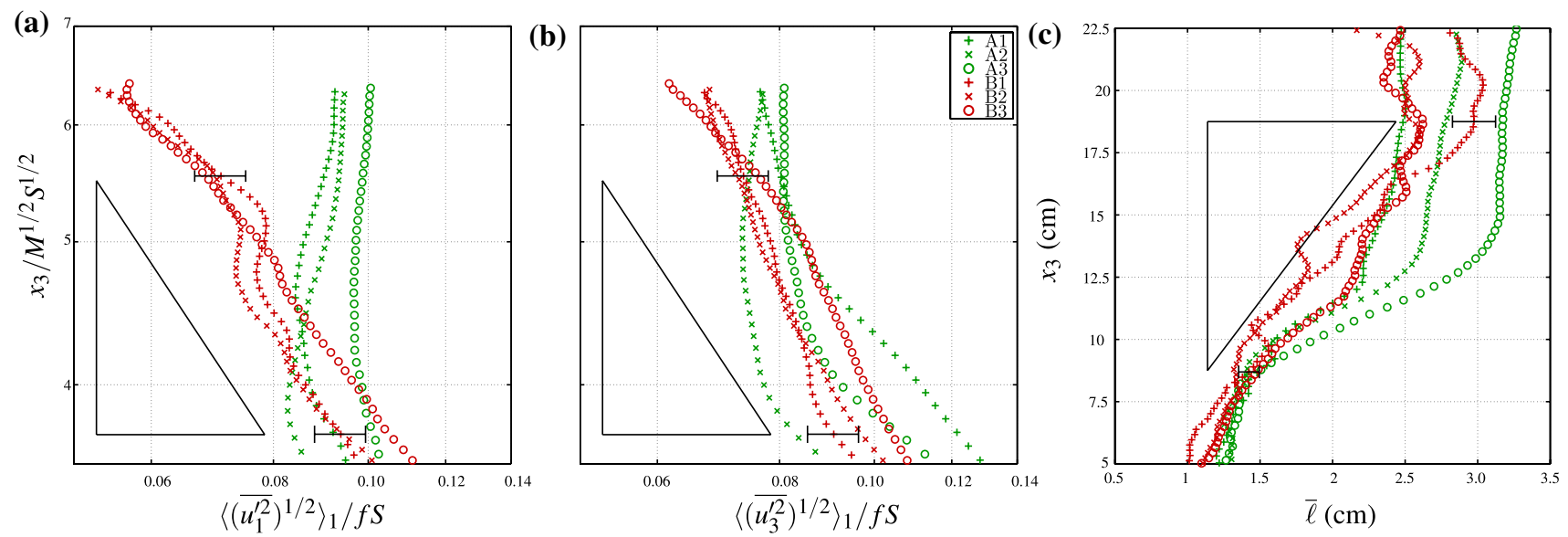

Fig. 10 a, b Spatially averaged (in the $x_{1}$-direction) measurements of the rms velocity components $\left\langle\left(\overline{u_{1}^{\prime 2}}\right)^{1 / 2}\right\rangle_{1}$ and $\left\langle\left(\overline{u_{3}^{\prime 2}}\right)^{1 / 2}\right\rangle_{1}$ plotted against $x_{3}$; the quantities have been scaled in accordance with Eq. (1). The slope in each plot shows the spatial decay $\propto x_{3}^{-1}$. (c) Computed values of the time-averaged integral length scale, $\bar{\ell}$ plotted against normal-

conditions the turbulent transport is a dominant term. However, the data in Fig. 4 show that, with no inner box installed, the time-averaged mean flow is not negligible in the TDR, which contradicts the underlying assumptions, resulting in a poor fit; previous studies have shown that a significant mean flow can reduce the exponent of the spatial decay of $\left(\overline{u_{1}^{\prime 2}}\right)^{1 / 2}$ and $\left.\overline{\left(u_{3}^{\prime 2}\right.}\right)^{1 / 2}$ from approximately -1 to -0.5 (Hopfinger and Toly 1976). In contrast, with the inner box installed in the 'optimal' position (Experiments B1-B3) the reduction in mean-flow intensity within the turbulent-diffusive region results in turbulence that is in accordance with the underlying approximations made, such that the spatial decay of $\overline{\left(u_{1}^{\prime 2}\right)^{1 / 2}}$ and $\left.\overline{\left(u_{3}^{\prime 2}\right.}\right)^{1 / 2}$ is well-described by Eq. (1).

The velocity measurements were also used to estimate the longitudinal integral length scale. We followed the approach used previously (Kit et al. 1997; McCorquodale and Munro 2017) by defining the integral length scale as the integral of the autocorrelation function of $u_{1}^{\prime}\left(x_{1}, x_{3}, t\right)$, over the spatial lag up to which the autocorrelation function first crosses zero. The autocorrelation function, at spatial lag $\chi$, is defined as

$r_{u}\left(x_{3}, t ; \chi\right)=\frac{c_{u}\left(x_{3}, t ; \chi\right)}{c_{u}\left(x_{3}, t ; \chi=0\right)}$,

where

$c_{u}\left(x_{3}, t ; \chi\right)=\frac{1}{\zeta} \int_{-\zeta / 2}^{\zeta / 2} u_{1}^{\prime}\left(x_{1}, x_{3}, t\right) u_{1}^{\prime}\left(x_{1}+\chi, x_{3}, t\right) \mathrm{d} x_{1}$

Recall that $\zeta$ is used to denote the half-width of the outer box for Experiments A1-A3 or the half-width of the inner box for Experiments B1-B3; that is, Eq. (3b) is an integral ised distance below the grid $x_{3}$. The slope shows the Eq. (1c) with empirical constant $C_{2}=0.13$. The data for all conditions are shown separately [see legend in (b), which applies to all plots]. In each plot two error bars are shown for condition B1 only; these error bars are representative of all conditions

over the central region of the tank. The time-averaged integral length scale is then given by

$\bar{\ell}\left(x_{3}\right)=\frac{1}{T} \int_{0}^{T} \int_{0}^{\chi_{0}} r_{u}\left(x_{3}, t ; \chi\right) \mathrm{d} \chi \mathrm{d} t$

where $\chi_{0}$ denotes the spatial lag at which the autocorrelation function first becomes zero, and $T$ is the sampling period (which, recall, here was $240 \mathrm{~s}$ ). The corresponding estimates for $\bar{\ell}$ are shown in Fig. 10(c), plotted against the vertical coordinate $x_{3}$. (For reference we recall that the turbulent-diffusion region is given by $x_{3} \gtrsim 2.5 M=12.5 \mathrm{~cm}$.) Also shown are error bars, indicating the uncertainty within measured values of $\bar{\ell}$, computed using the same approach used to estimate uncertainty in measurements of $\left\langle\left(u_{1}^{\prime 2}\right)^{1 / 2}\right\rangle_{1}$ and $\left\langle\left(\overline{u_{3}^{\prime 2}}\right)^{1 / 2}\right\rangle_{1}$. These data show that with the inner box installed in the 'optimal' position (i.e. Experiments B1-B3) the growth of the longitudinal integral length scales are welldescribed by Eq. (1c) using a value of $C_{2} \approx 0.13$, which is consistent with previously reported values (Thompson and Turner 1975; De Silva and Fernando 1994; Kit et al. 1997). However, with no inner box installed (i.e. Experiments A1-A3) Eq. (1c) is a poor fit for the measured values of the longitudinal integral length scale.

\section{Conclusions}

We have presented experimental data describing a mechanism for the inception of mean flow in OGT, the means by which the magnitude of the mean flow can be reduced by use of an 'inner box' and the corresponding effect this has 
on the structure of the turbulence produced. Visualisation of the mean flow close to an oscillating grid, using 2D PIV data, indicates that the grid generates a regular structure of jets (away from the tank side walls) that break down to promote turbulence, confirming the recognised structure of OGT. However, a combination of grid edge effects and sidewall effects (viscous and no penetration) cause a deflection, and subsequent merging in the boundarynormal direction, of the jets produced below the perimeter region of the grid. These merged outer jets are more intense than those produced at the centre of the grid and give rise to large-scale circulations within the container.

Installation of an open-ended inner box, positioned symmetrically below the grid with its sidewalls equidistant between the two outermost grid intersections and with its top edge $1 \mathrm{~cm}$ below the bottom of the grid's oscillation, was shown to inhibit the formation of the merged outer jets and reduce mean flow velocities within the inner box. Remaining sources of mean flow within the inner box are sufficiently weak that turbulent fluctuations are the dominant component of the flow; measurements indicated that the 'mean flow intensity' (the ratio of the mean flow velocity component to the rms velocity component) was reduced from approximately 3.5 for experiments with no inner box installed to approximately 0.6 when the inner box was installed.

Analysis of the spatial distribution of turbulent energy and turbulent energy flux indicates that close to the sidewalls of the container OGT is strongly anisotropic and inhomogeneous. However, the correct installation of the inner box results in turbulence that is a good approximation to homogeneous and isotropic conditions (i.e., ${\overline{u_{1}^{\prime 2}}}^{2} \approx{\overline{u_{3}^{\prime 2}}}^{2} \approx$ constant), on horizontal planes parallel to the grid, over the central $50 \%$ of the inner box's width. Data are also presented to evaluate whether the installation of an inner box results in turbulence, within the TDR, that is in good accordance with the widely used idealised model. Results indicate that the degree of isotropy, the spatial decay of the rms turbulent velocity components and integral length scale are well-described by the standard model (Eq. 1) when the inner box is installed, but that the intense mean flow in experiments conducted with no inner box installed can result in turbulence for which Eq. (1) is a poor description.

We anticipate that this simple amendment to reduce the mean flow present in OGT can be readily used in future work that utilises OGT to isolate the effects of turbulent fluctuations from those of the mean flow.

Acknowledgements The authors acknowledge Jim Meakin and Steve Lawton for technical support with the experimental apparatus.
Open Access This article is distributed under the terms of the Creative Commons Attribution 4.0 International License (http://creativeco mmons.org/licenses/by/4.0/), which permits unrestricted use, distribution, and reproduction in any medium, provided you give appropriate credit to the original author(s) and the source, provide a link to the Creative Commons license, and indicate if changes were made.

\section{References}

Al-Homoud A, Hondzo M (2007) Energy dissipation estimates in oscillating grid setup: LDV and PIV measurements. Environ Fluid Mech 7:143-158

Bennett SJ, Hou Y, Atkinson JF (2014) Turbulence suppression by suspended sediment within a geophysical flow. Environ Fluid Mech 14:771-794

Brumley BH, Jirka GH (1987) Near-surface turbulence in a grid-stirred tank. J Fluid Mech 183:235-263

Brunk BK, Koch DL, Lion LW (1998) Observations of coagulation in isotropic turbulence. J Fluid Mech 371:81-107

Cheng NS, Law AWK (2001) Measurements of turbulence generated by oscillating grid. J Hydraul Eng 127:201-208

Dalziel SB (2006) Digiflow user guide: http://www.damtp.cam.ac.uk/ lab/digiflow/. Accessed July 2016

De Silva IPD, Fernando HJS (1994) Oscillating grids as a source of nearly isotropic turbulence. Phys Fluids 6:2455-2464

De Silva IPD, Fernando HJS (1998) Experiments on collapsing turbulent regions in stratified fluids. J Fluid Mech 358:29-60

Dickinson SC, Long RR (1983) Oscillating-grid turbulence including effects of rotation. J Fluid Mech 126:315-333

Dohan K, Sutherland BR (2002) Turbulence time scales in mixing box experiments. Exp Fluids 33:709-719

Drayton MJ (1993) Eularian and lagrangian studies of inhomogeneous turbulence generated by an oscillating grid. Ph.D. thesis, King's College, University of Cambridge

Fernando HJS, De Silva IPD (1993) Note on secondary flows in oscillating grid, mixing box experiments. Phys Fluids 5:1849-1851

Hannoun IA, Fernando HJS, List EJ (1988) Turbulence structure near a sharp density interface. J Fluid Mech 189:189-209

Honey RE, Hershberger R, Donnelly RJ, Bolster D (2014) Oscillating-grid experiments in water and superfluid helium. Phys Rev E 89(053):016

Hopfinger BJ, Linden PF (1982) Formation of thermoclines in zeromean-shear turbulence subjected to a stabilizing buoyancy flux. J Fluid Mech 11:157-173

Hopfinger BJ, Toly JA (1976) Spatially decaying turbulence and its relation to mixing across density interfaces. J Fluid Mech 78:155-175

Hopfinger EJ, Browand FK, Gagne Y (1982) Turbulence and waves in a rotating tank. J Fluid Mech 125:505-534

Hunt JCR, Graham JMR (1978) Free-stream turbulence near plane boundaries. J Fluid Mech 84:209-235

Huppert HE, Turner JS, Hallworth MA (1995) Sedimentation and entrainment in dense layers of suspended particles stirred by an oscillating grid. J Fluid Mech 289:263-293

Kinzel M, Holzner M, Lüthi B, Tropea C, Kinzelbach W, Oberlack M (2009) Experiments on the spreading of shear-free turbulence under the influence of confinement and rotation. Exp Fluids 47:801-809

Kit E, Fernando HJS, Brown JA (1995) Experimental examination of Eulerian frequency spectra in zero-mean-shear turbulence. Phys Fluids 7(5):1168-1170

Kit ELG, Strang EJ, Fernando HJS (1997) Measurement of turbulence near shear-free density interfaces. J Fluid Mech 334:293-314 
Lucas S, Moulin F, Guizien K (2016) Oscillating grid mesocosm for studying oxygen dynamics under controlled unsteady turbulence. Limnol Oceanogr Methods 14:1-13

McCorquodale MW (2017) Interaction between oscillating-grid turbulence and a solid impermeable boundary. Ph.D. thesis, University of Nottingham

McCorquodale MW, Munro RJ (2017) Experimental study of oscillating-grid turbulence interacting with a solid boundary. J Fluid Mech 813:768-798

McCorquodale MW, Munro RJ (2018) Analysis of intercomponent energy transfer in the interaction of oscillating-grid turbulence with an impermeable boundary. Phys Fluids 30:015105

McDougall TJ (1979) Measurements of turbulence in a zero-meanshear mixed layer. J Fluid Mech 94:409-431

McGrath JL, Fernando HJS, Hunt JCR (1997) Turbulence, waves and mixing at shear-free density interfaces. part 2. laboratory experiments. J Fluid Mech 347:235-261

McKenna SP, McGillis WR (2004) Observations of flow repeatability and secondary circulation in an oscillating grid-stirred tank. Phys Fluids 16(9):3499-3502

Nokes RI (1988) On the entrainment rate across a density interface. J Fluid Mech 188:185-204

\section{Affiliations}

\section{Mark W. McCorquodale ${ }^{1,2}$ (D) . R. J. Munro ${ }^{1}$ (D}

\section{R. J. Munro}

rick.munro@nottingham.ac.uk

1 Faculty of Engineering, University of Nottingham, Nottingham NG7 2RD, UK
Orlins JJ, Gulliver JS (2003) Turbulence quantification and sediment resuspension in an oscillating grid chamber. Exp Fluids 34:662-677

Shy SS, Tang CY, Fann SY (1997) A nearly isotropic turbulence generated by a pair of vibrating grids. Exp Therm Fluid Sci 14:251-262

Thompson SM, Turner JS (1975) Mixing across an interface due to turbulence generated by an oscillating grid. J Fluid Mech 67:349-368

Turner JS (1968) The influence of molecular diffusivity on turbulent entrainment across a density interface. J Fluid Mech 33(4):639-656

Variano EA, Bodenschatz E, Cowen EA (2004) A random synthetic jet array driven turbulence tank. Exp Fluids 37:613-615

Wan Mohtar WHM, Munro RJ (2013) Threshold criteria for incipient sediment motion on an inclined bedform in the presence of oscillating-grid turbulence. Phys Fluids 25:015103

Publisher Note Publisher's Note Springer Nature remains neutral with regard to jurisdictional claims in published maps and institutional affiliations.

2 Present Address: Department of Meteorology, University of Reading, Reading, UK 